\title{
Embedding of Real Varieties and their Subvarieties into Grassmannians
}

\author{
M.A. BUCHNER
}

\begin{abstract}
Given a compact affine nonsingular real algebraic variety $X$ and a nonsingular subvariety $Z \subset X$ belonging to a large class of subvarieties, we show how to embed $X$ in a suitable Grassmannian so that $Z$ becomes the transverse intersection of the zeros of a section of the tautological bundle on the Grassmannian.
\end{abstract}

In [2] Bochnak and Kucharz prove the following characterization of a compact nonsingular algebraic hypersurface $Z$ in a compact affine nonsingular real algebraic variety $X$ : There is an algebraic embedding $f: X \rightarrow R P^{n}$ (for some $n$ ) and a projective hyperplane $H \subset R P^{n}$ transverse to $f(X)$ such that $H \cap f(X)=f(Z)$. This fact (or rather a closely related statement about strongly algebraic real line bundles) plays a crucial role in their construction of algebraic models $Y$ of a compact, connected, smooth manifold $M$ of dimensions $m \geq 3$ such that

Supported by Nato Grant CRG 930238.

1991 Mathematics Subject Classification: 14P05

Servicio publicaciones Univ. Complutense. Madrid, 1995. 
the algebraic homology elements in $H^{1}(Y, Z / 2)=H^{1}(M, Z / 2)$ form a prescribed subgroup $G \subset H^{1}(M, Z / 2)$. If we wish to extend this result to subgroups of $H^{k}(M, Z / 2)$ for $k>1$ it seems desirable, as a first step, to extend the above characterization of hypersurfaces to subvarieties of higher codimension.

Let $G_{n, k}(R)$ denote the Grassmannian of $k$-planes in $R^{n}$. Let $\gamma_{n, k}$ denote the universal bundle over $G_{n, k}(R)$. For definitions and results concerning real varieties, strongly algebraic vector bundles etc. see [1].

Theorem 1. Let $X$ be a compact affine nonsingular real algebraic variety. Let $\zeta$ be a strongly algebraic real vector bundle over $X$ of rank $k$. Let $\sigma$ be a regular section of $\zeta$ transverse to the zero section. Let $Z=\sigma^{-1}(0)$. Then

(i) There exists a regular embedding $f: X \rightarrow G_{n, k}(R)$ for suitable $n$ such that $\zeta$ and $f^{*}\left(\gamma_{n, k}\right)$ are isomorphic.

(ii) There exists a regular section $s$ of $\gamma_{n, k}$ such that $s$ is transversal to the zero section and $s^{-1}(0) \cap f(X)=f(Z)$ (the intersection $s^{-1}(0) \cap$ $f(X)$ being transverse intersection).

Proof. We can assume that $X$ is a subvariety of real projective $q$ space $R P^{q}$ for some $q$. By theorem 12.1.7 of [1] there is a regular map $g: X \rightarrow G_{\ell, k}(R)$ (for suitable $\ell$ ) such that $g^{*}\left(\gamma_{\ell, k}\right)$ and $\zeta$ are isomorphic. Let $G_{\ell, k}(C)$ denote the Grassmannian of complex $k$-planes in $C^{\ell}$ and $\gamma_{\ell, k}^{C}$ the corresponding universal complex bundle. Let $X_{C}$ denote the complexification of $X$ in $C P^{q}$. Then $g$ extends to a regular map $\tilde{g}$ : $U \rightarrow G_{\ell, k}(C)$ where $U \subset X_{C}$ is a Zariski open set containing $X$. We can assume $U$ and $\bar{g}$ are defined over $R$. By resolution of singularities we can find a complex nonsingular subvariety $Y$ of some complex projective space $C P^{m}$ with $Y$ defined over $R$ and a regular map (defined over $R$ ) $\tau: Y \rightarrow X_{C}$ where $\tau$ is the composition of a sequence of blowings-up with real centers outside $U$ such that $\tilde{g} \circ \tau$ extends to a regular map on $Y$. Denote this extension by $h$. To simplify notation we identify $X$ with $\tau^{-1}(X)$. Then $h^{*}\left(\gamma_{\ell, k}^{C}\right)$ is a bundle defined over $R$ and $h^{*}\left(\gamma_{\ell, k}^{C}\right) \mid X$ is isomorphic to $\zeta \otimes C$.

Now, for $E \rightarrow M$ a holomorphic vector bundle of rank $k$ over the compact complex manifold $M$, let $H^{0}(M, E)$ denote the space of holomorphic sections. Denote the dimension of $H^{0}(M, E)$ by $n$. Let 
$i_{E}(x)=\{$ sections vanishing at $x\}$. Assume that each fiber of $E$ is generated by global sections. Then identifying $H^{0}(M, E)$ with $C^{n}$ we see that $i_{E}$ maps $M$ to $G_{n, n-k}(C) \simeq G_{n, k}(C)$. If $F \rightarrow M$ is a positive holomorphic line bundle then for $p$ sufficiently large $i_{E \otimes F^{P}}$ is an embedding of $M$ into $G_{n, k}(C)$ where, now, $n=\operatorname{dim}_{C} H^{0}\left(M, E \otimes F^{P}\right)$ and $i_{E \otimes F^{P}}^{*}\left(\gamma_{n, k}^{C}\right)$ is isomorphic to the bundle $E \otimes F^{P} \rightarrow M$. Apply this to $E \rightarrow M$ replaced by $h^{*}\left(\gamma_{\ell, k}^{C}\right)$ (so $M$ is replaced by $Y$ ) and $F$ replaced by $\gamma_{m, 1}^{C} \mid Y$. In this case $i_{E \otimes F^{P}}$ is a regular map defined over $R$. Abbreviating $i_{E \otimes F}$, by $i$, we can write

$$
i^{*}\left(\gamma_{n, k}^{C}\right) \simeq h^{*}\left(\gamma_{\ell, k}^{C}\right) \otimes\left(\gamma_{m, 1}^{C} \mid Y\right)^{p}
$$

(as complex bundles). We now restrict both sides to $X$ and obtain

$$
(i \mid X)^{*}\left(\gamma_{n, k}\right) \otimes C \simeq(\zeta \otimes C) \otimes\left(\left(\gamma_{m, 1} \mid X\right) \otimes C\right)^{p}
$$

and hence

$$
(i \mid X)^{*}\left(\gamma_{n, k}\right) \simeq \zeta \otimes\left(\gamma_{m, 1} \mid X\right)^{p}
$$

We can assume $p$ is even. Then $\left(\gamma_{m, 1} \mid X\right)^{p}$ is topologically trivial. Hence $(i \mid X)^{*}\left(\gamma_{n, k}\right)$ is topologically and hence algebraically isomorphic to $\zeta$. This completes the proof of (i) with $f=i \mid X$.

To simplify notation we now identify $X$ with $f(X)$ and $\zeta$ with $\gamma_{n, k} \mid X$. Let $s_{1}, \ldots, s_{n}$ be sections of $\gamma_{n, k}$ (over $G_{n, k}(R)$ ) spanning the fiber at each point of $G_{n, k}(R)$. Write $\sigma=\Sigma \lambda_{i}\left(s_{i} \mid X\right)$ where $\lambda_{i}$ are regular real-valued functions on $X$. Let $\tilde{\lambda}$ be a regular extension of $\lambda_{i}$ to $G_{n, k}(R)$. Let $\phi$ be a regular real-valued function on $G_{n, k}(R)$ such that $\phi^{-1}(0)=Z\left(=\sigma^{-1}(0)\right)$. For $t=\left(t_{1}, \ldots, t_{n}\right)$, define $s_{t}=\Sigma_{i=1}^{n}\left(\tilde{\lambda}_{i}+t_{i} \phi^{2}\right) s_{i}$. We can find $t$ (suitably small) so that $s_{t}$ is transverse to the zero section, $s_{t}^{-1}(0)$ is transverse to $X$ and $s_{t}^{-1}(0) \cap X=$ $\sigma^{-1}(0)(=Z)$. This completes the proof of (ii).

\section{References}

[1] Bochnak, J., Coste, M. and Roy, M.-F., Géométrie algébrique reelle. Ergebnisse der Math. Vol. 12, Berlin, Heidelberg, New York: Springer 1987. 
[2] Bochnak, J. and Kucharz, W., Algebraic models of smooth manifolds. Invent. Math. Vol. 97, pp. 585-611 (1989).

Department of Mathematics and Statistics

Recibido: 19 de Abril de 1994

University of New Mexico

Albuquerque, New Mexico 87131 